\title{
MAGIC and the very high energy gamma-ray sky
}

\author{
Bernardo Fraga for the MAGIC Collaboration* \\ Centro Brasileiro de Pesquisas Físicas, CBPF, Rua Dr. Xavier Sigaud 150, Brazil \\ E-mail: bernardoecbpf.br
}

\begin{abstract}
MAGIC is a system of two $17 \mathrm{~m}$ Cherenkov telescopes for very high energy gamma-ray and cosmic-ray astrophysics and currently one of the best performing in the field. Through the discovery of more than 50 extragalactic and galactic sources including blazars, radiogalaxies, pulsars, supernovae remnants and binary systems (among other types) a broad range of topics can be studied: accretion to black holes, relativistic jets, shocks and their connection to the acceleration of cosmic rays. In addition, MAGIC tries to answer fundamental physics questions, such as the search for dark matter. In this talk I will discuss some selected highlights from MAGIC, especially the recent detection of very high energy gamma-rays from the blazar TXS 0506+056, which could potentially be the source of the high-energy extragalactic neutrino recently detected by Icecube.
\end{abstract}

International Conference on Black Holes as Cosmic Batteries: UHECRs and Multimessenger Astronomy BHCB2018

12-15 September, 2018

Foz du Iguazu, Brasil

${ }^{*}$ Speaker. 


\section{Introduction}

Built in the Canary Island of La Palma in 2004, MAGIC operated as a single telescope until 2009, when the construction of MAGIC-II was finished and it started operating in stereo mode. It detects the Cherenkov light produced by extended air showers initiated when $\gamma$-rays (or cosmic rays) interact with the molecules in the atmosphere. This light is captured as an image by the cameras and an analysis is done to reject the hadronic showers and reconstruct the true energy and direction of the photons. The energy threshold in stereoscopic mode is as low as $50 \mathrm{GeV}$, with a sensitivity of $0.66 \%$ of the Crab nebula above $220 \mathrm{GeV}$ and an angular resolution of 0.06 degrees at $1 \mathrm{TeV}$. Moreover, it can slew as fast as $7 \mathrm{deg} / \mathrm{sec}$, allowing it to quickly follow transients and trigger alerts. More details on the performance can be found on [1].

Although MAGIC achieves its best performance in dark time, operating only during moonless nights would severely limit the duty cycle. Observing under partial (or even full) moonlight involves reducing the Photomultipliers gain or using UV pass filters to block most of moonlight, and it can increase the duty cycle up to $40 \%$ [2]. The Crab nebula spectrum is recovered in different illumination conditions, reaching up to 30 times the dark sky background, with a sensitivity degradation of at most $80 \%$. Moreover, a non-standard analysis is needed, as the main effects of moonlight is an increase in the energy threshold and the systematics uncertainties of flux normalization.

Here I will present some selected MAGIC highlights from its 15 years of operations.

\section{Galactic Sources}

Since its discovery in the by the Whipple telescope in 1989 (the first detection of a TeV source), the Crab pulsar has been one of the main targets for VHE observatories. Despite the efforts, the mechanism for the pulsed emission is still under debate, with some models predicting either a super-exponential or an exponential cutoff at a few GeV. However, after $\approx 22$ hours of observation, MAGIC detected pulsed emission above $25 \mathrm{GeV}$ with no hint of cutoff[3]. This posed a serious challenge to models predicting a super exponential cutoff at a few $\mathrm{GeV}$, and it showed that the emission is likely to be originated farther from the neutron star. More insightful discoveries followed, such as the detection of the bridge emission[4] and the extension of the spectrum up to 400 $\mathrm{GeV}[5]$. In one of the latest results, MAGIC combined data from 2007 to 2014, for a total of 320 hours of observation time[6]; this is one of the deepest observations of one object performed by IACTs. With this dataset, MAGIC has detected for the first time pulsed emission from the Crab above $400 \mathrm{GeV}$, going up to $1.5 \mathrm{TeV}$ with no hint of a cutoff, as seen on Fig. 1. . Emission of pulsed photons in the $\mathrm{TeV}$ range favors inverse Compton scattering as opposed to synchro-curvature radiation as the emission mechanism, although it is still under debate where in the magnetosphere the upscattering occurs.

Pulsar Wind Nebulae (PWN) are the most numerous TeV source in our galaxy. 3C 58 is powered by one of the highest spin down pulsars known, but despite previous efforts by other VHE observatories (including MAGIC itself) it remained undetected. After some upgrades, MAGIC was finally able to confirm the detection[7], showing it to be the faintest PWN ever discovered and 


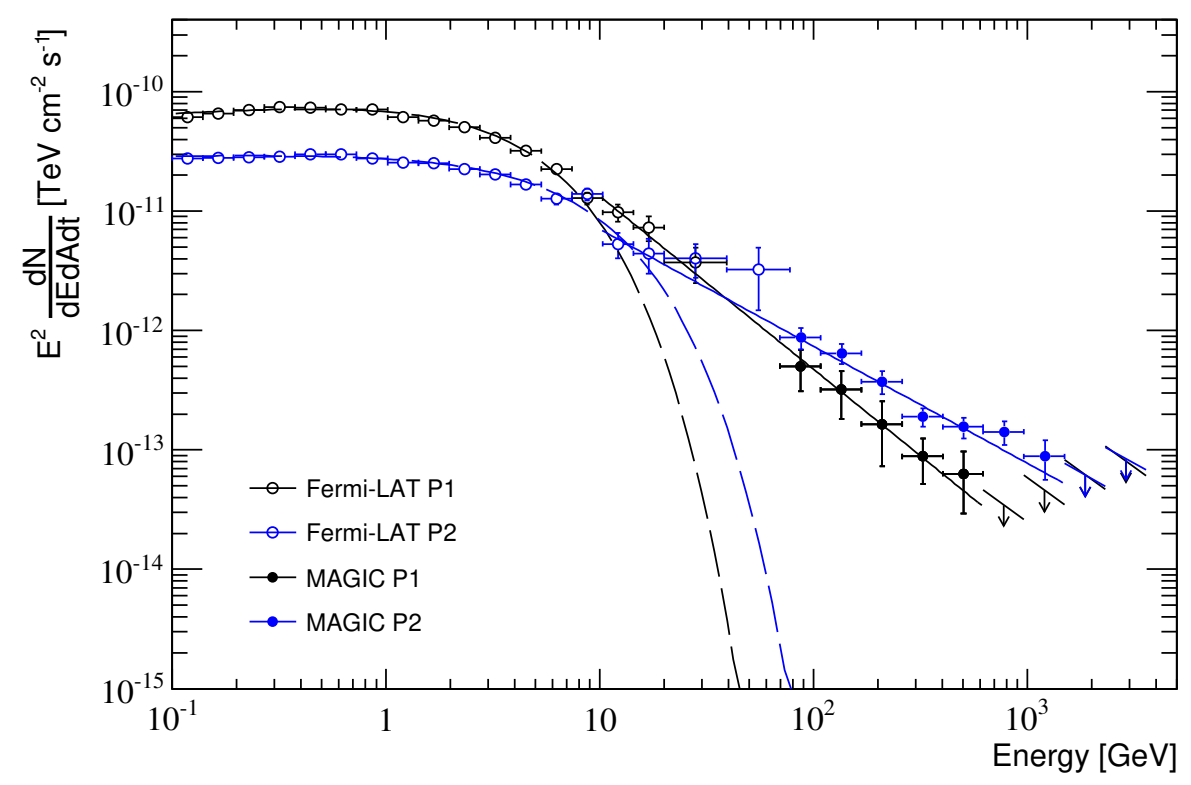

Figure 1: Phase-folded SED of the Crab pulsar emission peaks P1 and P2. Solid lines indicate the joint Fermi-LAT/MAGIC powerlaw fit above $10 \mathrm{GeV}$.

demonstrating how MAGIC can hunt for the faintest sources due to its excellent performance in the low energy range.

Supernovae remnants (SNRs) are thought to be accelerators of galactic cosmic rays up to the 'knee' (around $3 \mathrm{PeV}$ ), and in this process emit $\gamma$-rays. Cassiopeia A (Cas A) is one of the prime candidates to study this, since its age is known (300 years) and it was already studied both in radio as in x-rays, constraining essential parameters for modelling. MAGIC collected more than 160 hours of observation ( $\sim 73 \%$ of which during mootime), and measured the SED up to $8 \mathrm{TeV}$ [8]. It is best described by a power law with an exponential cutoff at $3.5 \mathrm{TeV}$. If the $\gamma$-rays are produced by hadronic processes, this result indicates that Cas $\mathrm{A}$ is not a PeVatron (PeV accelerator) at the present time.

\section{Dark Matter searches with MAGIC}

MAGIC is also a valuable dark matter (DM) probe, since $\gamma$-rays can be produced by annihilation or decay reactions in rich DM regions. Besides that, for several channels, the photon spectrum can peak at the $\mathrm{TeV}$ and specific features may allow us to differentiate a DM signal from purely astrophysical ones, and could provide clues to help the identification of the particle besides its detection.

One of the most promising targets for these studies are dwarf Spheroidal galaxies (dSphs), small satellite galaxies of the Milky Way that are composed mainly of DM and present very little stellar activity, limiting heavily the astrophysical contamination. MAGIC performed the longest exposure of a single dSph in Segue I between 2011 and 2013, accumulating almost 160 hours of 
observing time[9]. This provided the best constraints for annihilating DM above $100 \mathrm{GeV}$, and was published in the Particle Physics Booklet.

\section{Extragalactic Sources}

Due to its location in the Northern Hemisphere, MAGIC is more suited to search for extragalactic sources. It has detected up to now more than 40 AGNs (see Fig. 2) and the number is continually increasing. $\mathrm{TeV}$ photons can interact with the ultraviolet-infrared background radiation of the Extragalactic Background Light (EBL), producing pairs; these photons are therefore lost and the VHE spectrum of distant sources is heavily attenuated, making it extremely difficult to detect $\mathrm{TeV}$ sources at high redshift. However, the low energy threshold of MAGIC allows it to hunt for

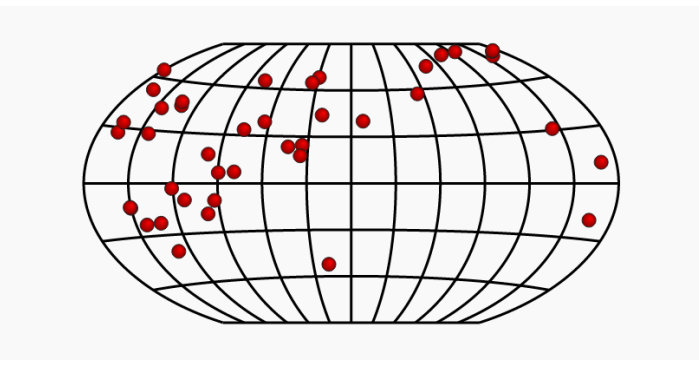

Figure 2: All Blazars detected by MAGIC. Taken from http://tevcat2.uchicago.edu/.

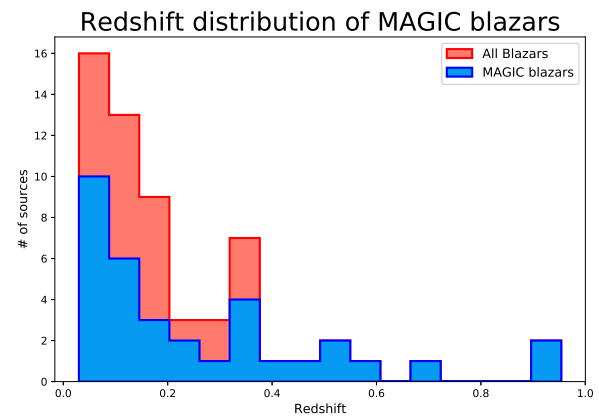

Figure 3: Redshift distribution of VHE detected blazars.

the farthest sources, and it is currently leading the effort to find high-z TeV blazars, as can be seen from Fig. 3.

MAGIC detected two of the most distant TeV blazars ever, at $\mathrm{z} \sim 1$ : PKS 1441+25[10] and QSO B0218+357[11], the first delayed VHE signal due to gravitational lensing. They helped extend EBL models to larger distances and improve the current ones.

One of the most important features of AGNs (and blazars in particular) is their variability, which could range from minutes to months and even years. Variability measurements can yield information about the size of the emitting region. MAGIC has detected minute-scale variability in different blazars, such as Mrk501[12] and PKS 1222+21[13], with flux-doubling times less than ten minutes. While this rapid variability can be explained in blazars by beaming effects, for Radio Galaxies (where the jet is at larger angles to the line of sight) it is more difficult. MAGIC has detected extreme variability in the Radio Galaxy IC 310 in one night where it flared[14] (see Fig. 4). The shortest variability timescale is $\sim 5$ minutes, corresponding to $20 \%-60 \%$ of the lightcrossing time.

Very Long Baseline Interferometry (VLBI) observations constrain the angle to the line of sight to be less than $20^{\circ}$ with a Doppler factor $\delta \sim 4$, putting it in the borderline between blazars and Radio Galaxies; however, in order for the emission region to be transparent to $\mathrm{TeV}$ photons, a much larger Doppler factor is needed. This tension between VHE and Radio measurements could be alleviated by assuming a different emission model (such as magnetospheric models, mini jet 


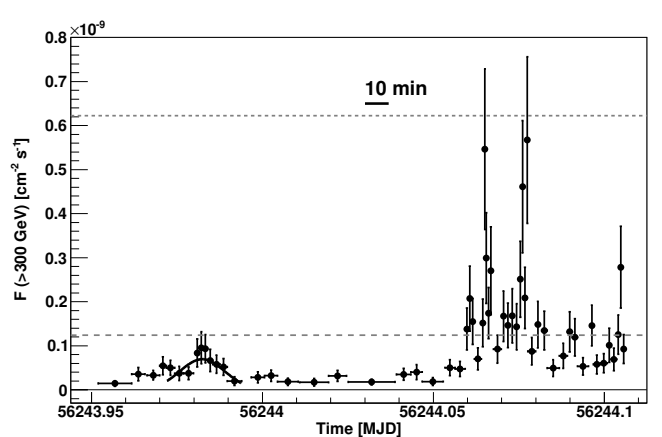

Figure 4: Lightcurve of IC 310.

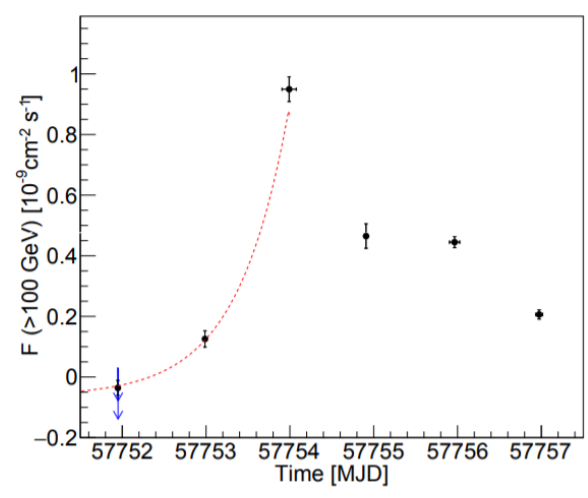

Figure 5: Lightcurve of NGC 1275 during the flare.

structures within the jet, jet-cloud interactions or others) or by assuming that the jet bends. The latter is disfavored since no perturbation on the jet flow direction is seen up to kiloparsec scale. NGC 1275[15] is another example where short scale variability was observed (although not so extreme, see Fig. 5) and where the Doppler factor (or viewing angle) is in disagreement with previous Radio measurements.

The only way to track changes of state and characterize long timescale variability is through multi-year, multiwavelength campaigns, and in fact MAGIC has participated in or developed several monitoring campaigns and Target of Opportunity $(\mathrm{ToO})$ programs based on external triggers.

One of the most observed blazars is Mrk 501. During a multiwavelength campaign in 2012 involving more than 25 instruments[16] including MAGIC, Swift, Fermi and others, it was observed that Mrk 501 behaved as an extreme HBL (EHBL, with a synchrotron peak above $10^{17} \mathrm{~Hz}$ ) and the VHE spectral index did not change with the state of the source. This suggests that the classification of a source as an EHBL might just be a temporary state rather than intrinsic behavior.

During the Mrk 421 campaign reported in [17], the source was observed with Swift, NuSTAR, Fermi-LAT, MAGIC and VERITAS. The flare shifted the peaks to higher energies, as well as hardened both x-ray and VHE spectrum. This could hint to a different electron population or a boost in the existing one due to some (yet) unknown mechanism. In yet another campaign on the same source[18], its $\gamma$-ray and x-ray activity was among the lowest ever, with the peaks shifted to lower energies. This could imply that HBLs can shift to IBLs, so that the classification based on the location of the synchrotron peak denotes a temporal state rather than a permanent feature, as was observed with Mrk 501.

One of the key observational programs of MAGIC is GRBs, with more than 50 hours devoted to it per year. The alerts are received through the Gamma-ray Coordinate Network (GCN), and MAGIC was able to observe 97 of them so far. This is a case where MAGIC's fast slewing is a huge bonus, as 22 of those GRNs could be observed with less than 100 seconds delay; unfortunately, there's no firm detection so far.

\section{Multimessenger Astronomy}

In the recent years, the importance of Multimessenger astronomy is being more and more 
recognized. In this field, collaborative efforts are of extreme importance, and MAGIC has followed two main avenues:

- Gravitational waves: Finding the electromagnetic counterpart of a GW event is necessary to constrain the source's emission. MAGIC has an MoU with VIRGO/LIGO to identify and follow GW events since 2014. GW 151226 was first IACT follow-up of a GW event, with two others coming afterwards. The lack of precise coordinates for the GW, combined with the small field of view (and limited duty cycle) for IACTs makes such observations challenging, but there's considerable effort to alleviate these difficulties.

- Neutrinos: Neutrinos are linked to the production of VHE and UVHE cosmic rays, and several emission models predict neutrinos as their outcome, but so far neutrinos of astrophysical origin remain elusive. MAGIC is part of the Gamma-ray Follow-up program since 2012, with more than 30 hours invested in searching for EM counterparts of IceCube alerts.

Despite having followed nine of those up until the end of 2017, no astrophysical source was detected in the VHE that could be responsible for the neutrino emission. In September 22nd 2017, IceCube reported a neutrino event (IC 170922A [19]): its most probable energy was of $290 \mathrm{TeV}$, with a probability of astrophysical origin of $56 \%$, and at the time of the alert no additional excess of neutrinos was observed from the same direction. Moreover, it was in close proximity to a

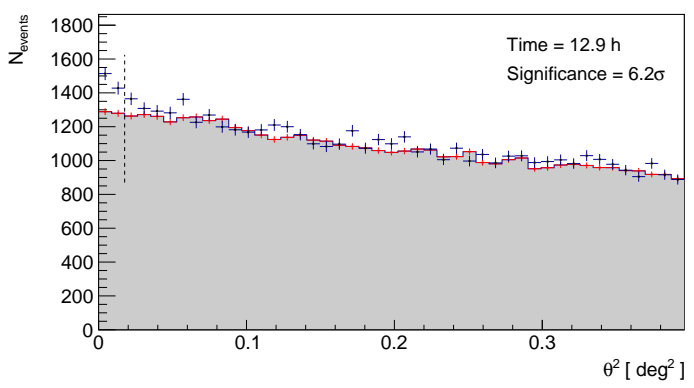

Figure 6: Distribution of angular distance from the nominal source position (points) and background estimation (shaded area).

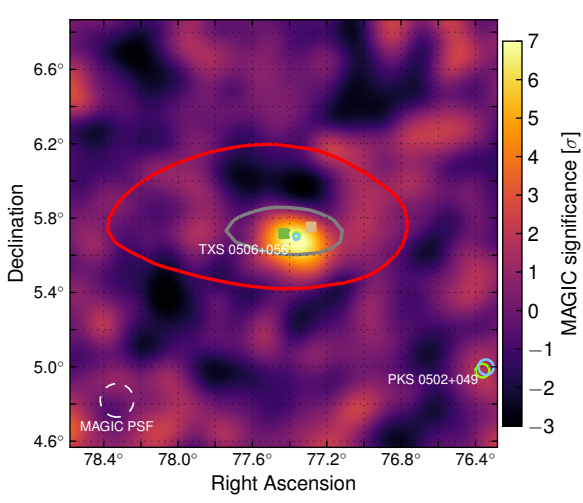

Figure 7: Position of IC 170922A overlaying the signal significance as observed by MAGIC. The tan square indicates the position reported in the initial alert and the green one indicates the final best-fitting position. Gray and red curves show $50 \%$ and $90 \%$ neutrino containment regions.

known Fermi blazar, TXS 0506+056. Following the alert, VHE observatories (H.E.S.S., MAGIC and VERITAS) tried to detect the source, but without success. However, after a report from Fermi of increased activity, MAGIC repointed and collected 13 hours of observations between September 28 and October 4, achieving a firm detection above 90 GeV[20], see Fig. 6 and Fig. 7.

Even though the nominal origin for the neutrino is only 6' away from the position of the blazar, it's still important to assess the probability that it was only a coincidence. This coincidence probability is a measure of the likelihood that a neutrino alert is correlated by chance with a flaring 
blazar, given the large number of known $\gamma$-ray sources and the modest number of neutrino alerts. This probability is calculated using simulated neutrino alerts and the light curve of Fermi-LAT sources, for different models of blazar-neutrino correlation. A likelihood ratio test is used to evaluate all models in a consistent manner, assuming that at least a some of the $\gamma$-ray flux is produced in the same hadronic interactions that produce the neutrinos. Three models for the $\gamma$-neutrino correlation were considered:

a) Neutrino flux correlated to the High Energy $\gamma$-ray flux. In this case neutrinos are more likely to be produced during hard and bright states of the source.

b) Neutrino flux correlated to variations of HE flux. Here, a weak or strong sources are equally likely be a neutrino source.

c) Neutrino flux correlated to VHE $\gamma$-ray flux. Hadronic acceleration up to a few $\mathrm{PeV}$ is necessary to explain the detected neutrino energy, VHE sources appear as likely progenitors.

For all these models, after accounting for all prior neutrino events, the results are similar: chance coincidence can be ruled out at $3 \sigma$ level and TXS $0506+056$ is the likely counterpart of IC 170922A.

After the initial detection, MAGIC continued observing it, collecting a total of 41 hours until November $2^{\text {nd }}[21]$. During this period TXS 0506+056 underwent two flare episodes, with a lower state in between; the spectrum can be modelled by a powerlaw, which is significantly steeper than the measured Fermi-LAT spectrum, and has no spectral variability. An intense multiwavelength

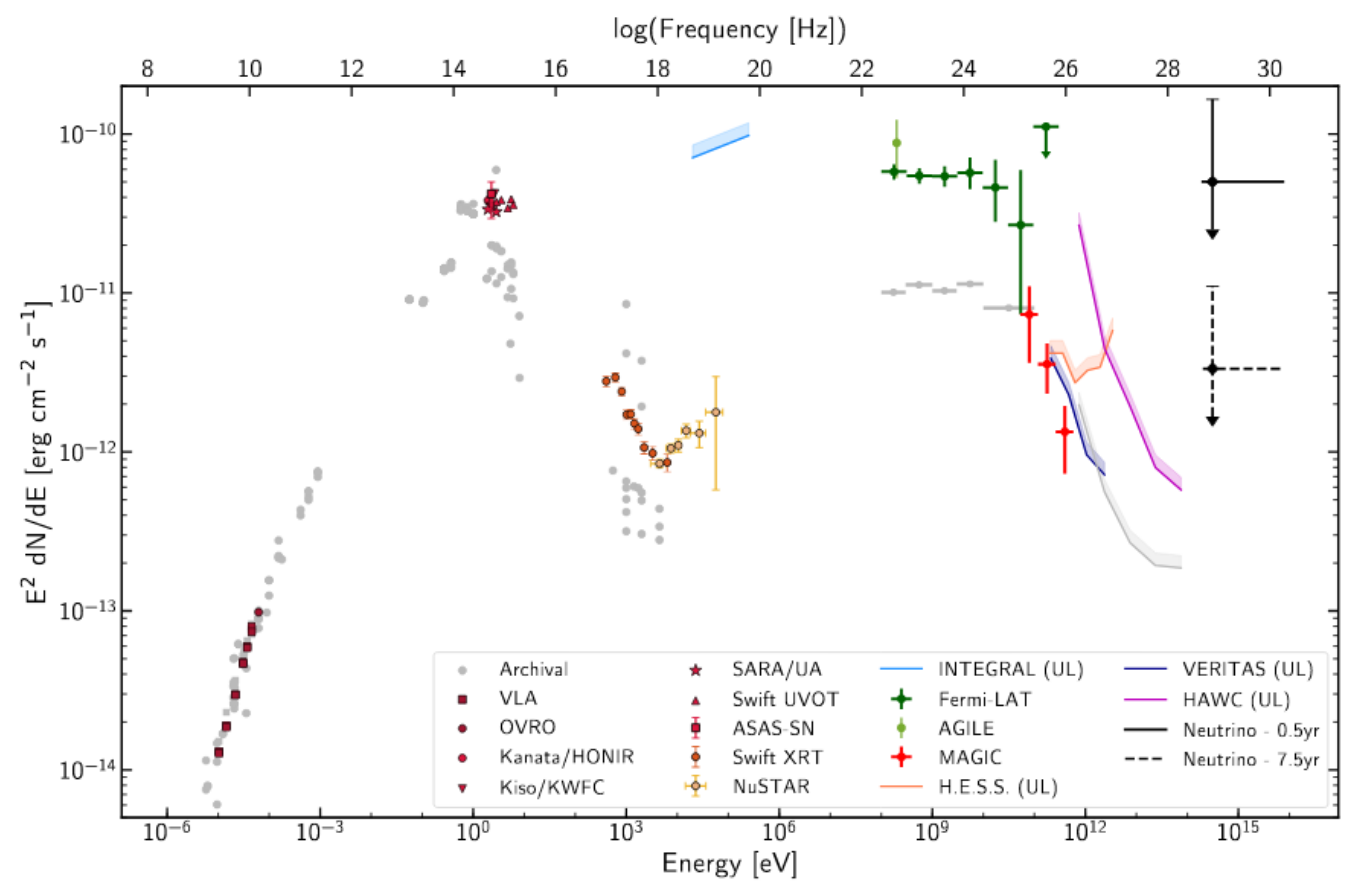

Figure 8: Multiwavelength SED of the blazar TXS 0506+056.

campaign was undertaken to better characterize TXS 0605+056 and build its Spectral Energy Distribution (SED), seen in Fig. 8.

Given the results above, one natural question arises: what is the mechanism by which BL Lacs can produce neutrinos? They can be produced through proton- $\gamma$ interactions in the jet, but 
in this case BL Lacs are disfavored for FSRQS due to the lower density of photon fields for the former. For the measured energy of the IceCube neutrino, protons with an energy of more than 6 $\mathrm{PeV}$ interacting with $\mathrm{UV}$ or soft $\mathrm{x}$-rays photons are necessary. A possible solution to this problem could come from structured jets[22], such as the spine-sheath model[23]: in these scenarios, the photon density from the sheath is seen boosted in the jet frame, providing the necessary density to produce neutrinos. The combined EM and neutrino emission can be interpreted in the jet-sheath model, with the steepening in the spectrum being the result of internal $\gamma \gamma$ absorption, as expected as a consequence of $p \gamma$ production of a $290 \mathrm{TeV}$ neutrino. Moreover, the inferred maximum proton energy can be as high as $1 \mathrm{EeV}$ in the comoving jet frame, high enough so that protons and/or heavy nuclei accelerated in blazars could make an important contribution to the Ultra High Energy Cosmic Rays (UHECRs)[21]. Unfortunately, with only a single neutrino event, stronger constraints are not possible, future multimessenger observations could shed some light on the mechanisms of acceleration and production of UHECRs.

\section{Conclusion}

Through its 15 years of operation, MAGIC has made several important discoveries, and it is still a very active collaboration. From the discovery of pulsed emission from the Crab pulsar in the $\mathrm{TeV}$ regime to the detection of several high-redshift AGNs, MAGIC has contributed both to galactic and extragalactic astrophysics. Moreover, it also dedicates a good amount of time in search for Dark Matter and GRBs. Recently, with the firm detection of a blazar likely responsible for the emission of a neutrino, it has entered the multimessenger era and it will be a major player in the area in the forthcoming years.

\section{Acknowledgments}

We would like to thank the IAC for the excellent working conditions at the ORM in La Palma. We acknowledge the financial support of the German BMBF, DFG and MPG, the Italian INFN and INAF, the Swiss National Fund SNF, the European ERDF, the Spanish MINECO, the Japanese JSPS and MEXT, the Croatian CSF, and the Polish MNiSzW.

BF is supported by FAPERJ grants 202.687/2016 and 202.688/2016.

\section{References}

[1] J. Aleksić, S. Ansoldi, L. A. Antonelli, P. Antoranz, A. Babic, P. Bangale et al., The major upgrade of the MAGIC telescopes, Part II: A performance study using observations of the Crab Nebula, Astroparticle Physics 72 (2016) 76 [1409.5594].

[2] M. L. Ahnen, S. Ansoldi, L. A. Antonelli, C. Arcaro, A. Babić, B. Banerjee et al., Performance of the MAGIC telescopes under moonlight, Astroparticle Physics 94 (2017) 29 [170 400906 ].

[3] E. Aliu, H. Anderhub, L. A. Antonelli, P. Antoranz, M. Backes, C. Baixeras et al., Observation of Pulsed $\gamma$-Rays Above 25 GeV from the Crab Pulsar with MAGIC, Science 322 (2008) 1221 [0809.2998]. 
[4] J. Aleksić, S. Ansoldi, L. A. Antonelli, P. Antoranz, A. Babic, P. Bangale et al., Detection of bridge emission above $50 \mathrm{GeV}$ from the Crab pulsar with the MAGIC telescopes, A\&A 565 (2014) L12 [1402.4219].

[5] J. Aleksić, E. A. Alvarez, L. A. Antonelli, P. Antoranz, M. Asensio, M. Backes et al., Phase-resolved energy spectra of the Crab pulsar in the range of 50-400 GeV measured with the MAGIC telescopes, A\&A 540 (2012) A69 [1109.6124].

[6] S. Ansoldi, L. A. Antonelli, P. Antoranz, A. Babic, P. Bangale, U. Barres de Almeida et al., Teraelectronvolt pulsed emission from the Crab Pulsar detected by MAGIC, A\&A 585 (2016) A133 [1510.07048].

[7] J. Aleksić, S. Ansoldi, L. A. Antonelli, P. Antoranz, A. Babic, P. Bangale et al., Discovery of TeV $\gamma$-ray emission from the pulsar wind nebula 3C 58 by MAGIC, A\&A 567 (2014) L8 [1405.6074].

[8] M. L. Ahnen, S. Ansoldi, L. A. Antonelli, C. Arcaro, A. Babić, B. Banerjee et al., A cut-off in the TeV gamma-ray spectrum of the SNR Cassiopeia A, MNRAS 472 (2017) 2956 [1 707.01583 ].

[9] J. Aleksić, S. Ansoldi, L. A. Antonelli, P. Antoranz, A. Babic, P. Bangale et al., Optimized dark matter searches in deep observations of Segue 1 with MAGIC, JCAP 2 (2014) 008 [1312.1535].

[10] M. L. Ahnen, S. Ansoldi, L. A. Antonelli, P. Antoranz, A. Babic, B. Banerjee et al., Very High Energy $\gamma$-Rays from the Universe's Middle Age: Detection of the $z=0.940$ Blazar PKS 1441+25 with MAGIC, ApJL 815 (2015) L23 [1512.04435].

[11] M. L. Ahnen, S. Ansoldi, L. A. Antonelli, P. Antoranz, C. Arcaro, A. Babic et al., Detection of very high energy gamma-ray emission from the gravitationally lensed blazar QSO B0218+357 with the MAGIC telescopes, A\&A 595 (2016) A98 [1609.01095].

[12] J. Albert, E. Aliu, H. Anderhub, P. Antoranz, A. Armada, C. Baixeras et al., Variable Very High Energy $\gamma$-Ray Emission from Markarian 501, ApJ 669 (2007) 862 [astro-ph / 0702008 ].

[13] J. Aleksić, L. A. Antonelli, P. Antoranz, M. Backes, J. A. Barrio, D. Bastieri et al., MAGIC Discovery of Very High Energy Emission from the FSRQ PKS 1222+21, ApJL 730 (2011) L8 [1101. 464 5].

[14] J. Aleksić, S. Ansoldi, L. A. Antonelli, P. Antoranz, A. Babic, P. Bangale et al., Black hole lightning due to particle acceleration at subhorizon scales, Science 346 (2014) 1080 [1412 . 4936].

[15] MAGIC Collaboration, S. Ansoldi, L. A. Antonelli, C. Arcaro, D. Baack, A. Babić et al., Gamma-ray flaring activity of NGC1275 in 2016-2017 measured by MAGIC, A\&A 617 (2018) A91 [1806.01559].

[16] M. L. Ahnen, S. Ansoldi, L. A. Antonelli, C. Arcaro, A. Babić, B. Banerjee et al., The extreme HBL behaviour of Markarian 501 during 2012, ArXiv e-prints (2018) [1808.04300].

[17] J. Aleksić, S. Ansoldi, L. A. Antonelli, P. Antoranz, A. Babic, P. Bangale et al., Unprecedented study of the broadband emission of Mrk 421 during flaring activity in March 2010, A\&A 578 (2015) A22 [1412.3576].

[18] M. Baloković, D. Paneque, G. Madejski, A. Furniss, J. Chiang, M. Ajello et al., Multiwavelength Study of Quiescent States of Mrk 421 with Unprecedented Hard X-Ray Coverage Provided by NuSTAR in 2013, ApJ 819 (2016) 156 [1512.02235].

[19] I. Collaboration, GRB Coordinates Network/AMON Notices 50579430_130033, Sept., 2017.

[20] IceCube Collaboration, M. G. Aartsen, M. Ackermann, J. Adams, J. A. Aguilar, M. Ahlers et al., Multimessenger observations of a flaring blazar coincident with high-energy neutrino IceCube-170922A, Science 361 (2018) eaat1378 [1807.08816]. 
[21] S. Ansoldi, L. A. Antonelli, C. Arcaro, D. Baack, A. Babić, B. Banerjee et al., The Blazar TXS 0506+056 Associated with a High-energy Neutrino: Insights into Extragalactic Jets and Cosmic-Ray Acceleration, ApJL 863 (2018) L10 [1807 . 0430 0].

[22] G. Ghisellini, F. Tavecchio and M. Chiaberge, Structured jets in TeV BL Lac objects and radiogalaxies. Implications for the observed properties, A\&A 432 (2005) 401 [astro-ph/0406093].

[23] F. Tavecchio and G. Ghisellini, On the spine-layer scenario for the very high-energy emission of NGC 1275, MNRAS 443 (2014) 1224 [1404.6894]. 